\title{
The Gut Microbiome in Inflammatory Bowel Diseases: Diagnostic and Therapeutic Implications
}

\author{
Konrad Aden ${ }^{a}$ Wolfgang Reindl ${ }^{b}$

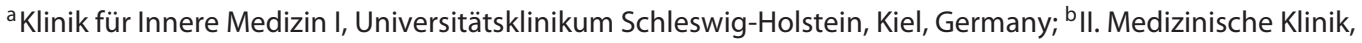 \\ Universitätsmedizin Mannheim, Medizinische Fakultät Mannheim der Universität Heidelberg, Mannheim, Germany
}

Keywords

Inflammatory bowel disease $\cdot$ Microbiota $\cdot$ Dysbiosis $\cdot$ Fecal microbial transfer

\section{Abstract}

The incidence of chronic inflammatory bowel diseases (IBD) is rising worldwide, and the interaction between the mucosal immune system and the intestinal microbiota is crucial for the understanding of these diseases. Due to new technologies, the data published on the intestinal microbiota has increased rapidly in the recent years. While many findings are descriptive, reporting associations between disease and microbial populations, recent advancement in technology made it possible to ask and answer more functional questions and to elucidate complex interactions between the intestinal microbiota and the mucosal immune system. In addition, first trials influenced the intestinal microbiota with the intention to treat IBD. This review summarizes aspects of the physiological function as well as the inflammation-induced changes of the gut microbiota and the association between the gut microbiota and pathogenesis in IBD. In addition, diagnostic and therapeutic options for treating IBD are reviewed.

\footnotetext{
(c) 2019 S. Karger AG, Base
}

\section{Introduction}

Inflammatory bowel diseases (IBD), including Crohn's disease (CD) and ulcerative colitis (UC), are characterized by chronic immune-mediated intestinal inflammation that is driven by a misled interplay of host-microbial interaction. The current concept of IBD pathogenesis is based on the theory of a disrupted intestinal barrier and a dysregulated immune response in a genetically susceptible host. These assumptions are underscored by early studies showing a strong clustering in families and with certain ethnicities. More recent studies showed a 15-50 times increased relative risk for siblings of a CD patient to also develop CD. In only $4 \%$ of all dizygotic twins both develop CD, yet in nearly $50 \%$ of all cases both monozygotic twins develop CD. In many of the families the pattern of the disease is also very similar, showing related complications, patterns of extra-intestinal manifestations, and organ involvement. IBD is a complex, polygenic disease with every patient having a very individual set of genes involved. Several genome-wide association studies and in silico meta-analysis of genetic data helped identify more than 200 genes associated with an increased risk to develop CD, UC, or both disease entities (for review, see Jostins et al. [1]). Altogether, genetics has led to the identification of various archetypical biological prin-

Konrad Aden

Klinik für Innere Medizin I, Universitätsklinikum Schleswig-Holstein

Arnold-Heller-Strasse 3

DE-24105 Kiel (Germany)

E-Mail k.aden@ikmb.uni-kiel.de 
ciples that contribute to disease pathophysiology on multiple independent cellular levels (e.g., T cells, B cells, and intestinal epithelial cells). These principles are as follows:

- defects in intestinal barrier function

- defects in autophagy

- disturbance of the innate immune system with an impaired phagocytic function

- hyper- and autoinflammatory reactions

- disturbed T- and B-cell activation and differentiation In addition to a variety of genetic factors, also environmental factors are essential for the development of IBD and are an important trigger for flairs of the disease. These environmental factors can be discriminated between factors originating in early childhood that increase the risk for developing IBD and other lifelong factors that increase the risk for flairs of already established disease (for review, see Lakatos [2]). The relevance of environmental factors is highlighted by studies on migrant populations. Moving from low-incidence areas to high-incidence areas, the incidence of IBD for the first generation born at the new place of residence is nearly identical to that of the resident population $[3,4]$. Obviously, this rapid equilibration in disease burden cannot be explained by changes in genetics. Currently, the highest prevalence rates for IBD are found in northern America, northern Europe, and Australia; however, countries with a rising socioeconomic status such as China or India currently see a steep rise of incidence in various immune-related disease, including IBD [5, 6]. Indisputably, nutrition is one of the strongest environmental factors influencing disease pathogenesis, yet it is difficult to pinpoint the proinflammatory potential of nutrition down to a single ingredient.

One common environmental factor in these areas is the western-style diet rich in sugar, fat, and protein. This diet has a strong and rapid influence on the intestinal microbiota and results in a microbiota distinctly different from the one seen with a plant-based diet [7]. Some smaller studies show a connection between fat- and proteinbased diet and IBD $[8,9]$; up to now large, prospective trials that show a direct connection between diet and IBD are missing. Another modulator of the intestinal microbiota associated with an increased risk for CD is the rising exposure to antibiotics [10]. Furthermore, the increase in Caesarian sections has been associated with an increased risk for IBD in the offspring due to a change in intestinal microbiota [11].

\section{Specific Changes in the Intestinal Microbiota Associated with IBD}

A key role of the gut microbiota in the pathophysiology of IBD has long been suggested; however, definitive cause-mechanism relationships in human IBD are cur- rently lacking. The predominant role of the gut microbiota in IBD derives from the association of microbial dysbiosis, defined as a decrease of microbial diversity and a selective overgrowth of potentially pathogenic microorganisms. Until today, several overlapping patterns of dysbiosis in IBD have emerged, including a reduction in biodiversity ( $\alpha$ - and $\beta$-diversity), a loss of "protective" bacteria of the Firmicutes phylum; e.g., Faecalibacterium prausnitzii [12], or concomitantly elevated "pathogenic" Gammaproteobacteria [13].

Due to the fact that IBD manifest itself most frequently in bowel segments with a high density of micro-organisms, a causal connection between the microbiota and IBD has been suspected for a long time [14]. As one histopathologic hallmark of CD is the epithelioid granuloma, many studies tried to prove a connection to an infection with atypical mycobacteria [15], yet up to now this hypothesis could not be proven. In contrast to this infection hypothesis, the role of the intestinal microbiota as an important driver of the intestinal inflammation in IBD has been proven by animal and clinical studies. A number of different mouse models did show significant improvement of symptoms or complete healing if the animals were treated with antibiotics $[16,17]$. In keeping with these experimental findings, patients suffering from active $\mathrm{CD}$ benefit from diverting ostomy or antibiotic treatment results by improvement of symptoms. Yet re-establishment of intestinal continuity or discontinuation of antibiotic therapy results in a rapid re-occurrence of intestinal inflammation [18-20]. Stool sampling in healthy adults reveals usually a spectrum of roughly 100 different bacterial species. This bacterial composition is specific for a given individual and usually very stable during the person's lifespan [21]. The typical western stool microbiota consists mainly of four phyla: Firmicutes, Bacteroidetes, Actinobacteriae, and Proteobacteria. Differential analysis of 16S rRNA of stool microbiota from patients with CU and $\mathrm{CD}$ revealed no typical population pattern for either disease, yet showed a typical reduction in diversity [22, 23]. The attempt to identify disease-specific differences in microbiota composition is hampered by the wide variety of different approaches to sampling and analysis and defining patient cohorts. Yet most studies find a reduction in Firmicutes and Bacteroidetes in CD patients' stool [24, 25] and mucosal biopsy samples [26]. In UC patients, these specific changes are less prominent [26]. Yet these changes in diversity and phyla compositions are specific for the context of intestinal inflammation as patients who have a pouch surgery after colectomy for familial adenomatous polyposis do not develop the same changes in local microbiota, leading to chronic pouchitis in some UC patients [27].

A detailed analysis beneath the "phylum layer" show the changes as described above, and also an increase in 
Gammaproteobacteria [26].These belong to the family of Enterobacteriacea and Pasteurellaceae that have been shown to be also increased in patients with CD [28]. The quantity of Clostridium group IV and XIV is decreased $[28,29]$. This is of functional relevance, since they are important for the production of butyrate, thus supporting the metabolism of epithelial cells and protecting the integrity of the epithelium and lowering the rate of apoptosis [30]. The reduction of butyrate-producing bacteria could also be demonstrated in patients with CU [31].

Currently, a bacterium from the group of Clostrdiae, Faecalibacterium prausnitzii, is in the center of attention, since a reduction of $F$. prausnitzii has been associated with a change in disease development. It could be shown that patients with $\mathrm{CD}$ and lower levels of $F$. prausnitzii have a higher risk of postoperative disease recurrence [12] and that patients with higher levels of this bacterium need to undergo surgery less often [29].

Despite the overwhelming evidence of an association of decreased gut microbial diversity with IBD, the precise role of dysbiosis in disease pathogenesis remains unclear. It remains an open question whether intestinal dysbiosis reflects the output of an overactivated inflammatory network of the host, which actively shapes the gut microbiota or rather reflects an independent layer of primary disease susceptibility that is shaped by host genetics and from the luminal site - by nutritional and environmental factors. Several studies, especially from animal models of intestinal inflammation, indicate that host factors (e.g., genetics, inflammatory tone) actively shape the intestinal microbiota and generate a transmissible colitogenic phenotype $[32,33]$. These studies point out the crucial relationship between host-mediated intestinal immune response mechanisms and gut microbial homeostasis and are further supported by various known IBD susceptibility variants in immune-related genes (e.g., NOD2, ATG16L1) that contribute to intestinal containment of gut microbiota [34].

In fact, changes in commensal microbiota have also been shown in patients carrying mutations of known IBD risk genes, such as NOD2, Atg161l, and Fucosyltransferase 2 (FUT2) [35, 36]. The modification of the innate and adaptive immune system triggered by these mutations not only influence the composition of the intestinal microbiota but also increases the vulnerability for intestinal pathogens [37, 38] and a loss of protective functions [39].

Importantly, many of the observed phenotypes can be recapitulated in mice carrying either full-body or conditional deletions (e.g., in intestinal epithelial cells) of IBD risk genes $[34,40,41]$. Hence, these studies further underline an important role of the intestinal epithelium in acting as the primary response layer of the mucosal immunity. On the other hand, gut dysbiosis might also reflect the response of a microbial community to environ- mental stress factors elicited from the inflamed mucosa. As an example, colonocyte metabolism in itself is intertwined with the metabolism of the gut microbiota as healthy colonocytes maintain an anaerobic condition, allowing the selection for obligate anaerobic organism. In contrast, inflamed colonocytes shift their metabolism towards aerobic conditions, allowing for the expansion of facultative aerobic organism contributing to gut dysbiosis [42].

\section{Functional Understanding of the Intestinal Microbiome in IBD}

Recent technological advances (comprehensive metabolomics, metagenomics, metaproteomics, and metatranscriptomic analyses are now feasible at relatively low cost per sample) have paved the way for a detailed mechanistic understanding on the functional consequences of gut dysbiosis in IBD. In that sense, the understanding of the microbiota's role in IBD has changed from the descriptive compositional information on the genus level into a more functional understanding of the contribution. In contrast to the anticipated potential of a mechanistic understanding of microbial metabolites in IBD dysbiosis, only few microbiota-generated metabolites affecting host physiology have been identified so far, demanding further study of the subject. These metabolites include for example secondary bile acid or trimethylamine-N-oxide, a product of microbial-host co-metabolism of nutrients such as phosphatidylcholine, choline, and L-carnitine, which are present in high-fat diets [43, 44]. An important identified pathway that has been implicated in IBD pathophysiology is the synthesis of shortchain fatty acids (SCFA), which originate from bacterial degradation of dietary fibers (e.g., inulin), and this pathway represents one of the few so far identified actionable metabolic pathways in IBD. It has been shown by various studies that disturbances in the microbial networks containing taxa that typically produce SCFA characterize treatment failure to conventional and biologic therapy $[45,46]$, rendering butyrate, a major microbiome-derived SCFA, as a potential candidate for metabolic intervention in IBD therapy [47]. However, previous attempts in reinstalling luminal SCFA production by butyrate enemas have not entirely proven efficacious in IBD therapy.

Mechanistically, the downstream role of SCFA on host biology are suggested to be mediated via GPR43, although recent data also indicate a potential role of SCFA in inducing innate lymphoid cell 3-dependent IL22 production and subsequent restoration of epithelial homeostasis [48]. Later studies linked a microorganism-derived metabolite (SCFA) with a well-studied immunological principle of pathobiont control (IL-22), 
and we and others have shown that mucosal IL-22 signalling influences epithelial proliferation and production of antimicrobial peptides, which in return contribute to the containment of gut microbiota, including viruses in IBD $[49,50]$.

\section{Microbiome Diagnostics and Therapeutics in IBD}

The acknowledgement of the gut microbiome as a potential reservoir of immunomodulatory metabolites that might actively shape host immune response has generated the concept of targeted microbiome editing as a therapeutic approach in IBD. However, current conceptual approaches have been made in murine studies, and the translational feasibility has not been shown yet. Recently, it has been shown that targeted inhibition of the molybdenum cofactor-dependent microbial respiratory pathways, which is selectively employed in anaerobic bacteria of the Enterobacteriaceae family during inflammation using the compound of tungsten, could ameliorate experimental colitis [51]. Although these findings are mindchanging in its conceptual design, it remains to be shown in the clinical future whether they provide a novel therapeutic approach in IBD. In contrast to experimental microbiome editing, the efficacy of fecal microbial transfer (FMT) in the treatment of UC has gained further evidence in clinical trials. Although clinical trials included relatively small patient numbers $(>100)$, these preliminary data indicate clinical efficacy of FMT in patients with UC [52, 53]. Importantly, detailed metabolomics and shotgun metagenomics analysis of the microbiota of FMT patients achieving clinical remission revealed an enrichment of Eubacterium halii and Roseburia inulivorans and increased levels of SCFA biosynthesis and secondary bile acids [54]. Hence, these data underscore that indeed specific metabolomic principles are crucially associated with clinical efficacy in IBD therapy. It remains to be shown whether pharmacological interception to enrich these pathways (e.g., SCFA synthesis) are efficacious in ameliorating disease activity or whether increased SCFA synthesis rather reflects the final homeostatic state of clinical remission.

\section{Conclusion}

Altogether, the current technological advances into a more functional understanding of the intestinal microbiome in actively contributing to mucosal immunology will provide future diagnostic and therapeutic entry points within the intestinal microbiota to optimize IBD therapy. Further research should therefore move away from the association of bacterial composition with presumable function and rather aim for a thorough mechanistic understanding to delineate the underlying host and microbial mechanisms that contribute to observed metabolitephenotype associations.

\section{Disclosure Statement}

K.A. received speaker fees from Janssen. W.R. received speaker fees from Abbvie, Janssen, Falk, and Takeda.

\section{References}

1 Jostins L, Ripke S, Weersma RK, Duerr RH, McGovern DP, Hui KY, et al.; International IBD Genetics Consortium (IIBDGC). Hostmicrobe interactions have shaped the genetic architecture of inflammatory bowel disease. Nature. 2012 Nov;491(7422):119-24.

2 Lakatos PL. Recent trends in the epidemiology of inflammatory bowel diseases: up or down? World J Gastroenterol. 2006 Oct; 12(38):6102-8.

3 Cosnes J, Gower-Rousseau C, Seksik P, Cortot A. Epidemiology and Natural History of Inflammatory Bowel Diseases. Gastroenterology. 2011 May;140(6):1785-94.

4 Ko Y, Butcher R, Leong RW. Epidemiological studies of migration and environmental risk factors in the inflammatory bowel diseases. World J Gastroenterol. 2014 Feb;20(5):123847.

5 Ng SC, Shi HY, Hamidi N, Underwood FE, Tang W, Benchimol EI, et al. Worldwide incidence and prevalence of inflammatory bowel disease in the 21st century: a systematic review of population-based studies. Lancet. 2018 Dec;390(10114):2769-78.
6 Molodecky NA, Soon IS, Rabi DM, Ghali WA, Ferris M, Chernoff $G$, et al. Increasing incidence and prevalence of the inflammatory bowel diseases with time, based on systematic review. Gastroenterology. 2012 Jan;142(1):46-54.e42.

7 Wu GD, Chen J, Hoffmann C, Bittinger K, Chen YY, Keilbaugh SA, et al. Linking longterm dietary patterns with gut microbial enterotypes. Science. 2011 Oct;334(6052):105-8.

8 Ananthakrishnan AN, Khalili H, Song M, Higuchi LM, Richter JM, Nimptsch K, et al. High School Diet and Risk of Crohn's Disease and Ulcerative Colitis. Inflamm Bowel Dis. 2015 Oct;21(10):2311-9.

9 Hou JK, Abraham B, El-Serag H. Dietary intake and risk of developing inflammatory bowel disease: a systematic review of the literature. Am J Gastroenterol. 2011 Apr;106(4): 563-73.

10 Ungaro R, Bernstein CN, Gearry R, Hviid A, Kolho KL, Kronman MP, et al. Antibiotics associated with increased risk of new-onset Crohn's disease but not ulcerative colitis: a meta-analysis. Am J Gastroenterol. 2014 Nov; 109(11):1728-38.
11 Bager P, Simonsen J, Nielsen NM, Frisch M. Cesarean section and offspring's risk of inflammatory bowel disease: a national cohort study. Inflamm Bowel Dis. 2012 May;18(5):857-62.

12 Sokol H, Pigneur B, Watterlot L, Lakhdari O, Bermúdez-Humarán LG, Gratadoux JJ, et al. Faecalibacterium prausnitzii is an anti-inflammatory commensal bacterium identified by gut microbiota analysis of Crohn disease patients. Proc Natl Acad Sci USA. 2008 Oct; 105(43):16731-6.

13 Rajca S, Grondin V, Louis E, Vernier-Massouille G, Grimaud JC, Bouhnik Y, et al. Alterations in the intestinal microbiome (dysbiosis) as a predictor of relapse after infliximab withdrawal in Crohn's disease. Inflamm Bowel Dis. 2014 Jun;20(6):978-86.

14 Kirsner JB. Historical aspects of inflammatory bowel disease. J Clin Gastroenterol. 1988 Jun;10(3):286-97.

15 Liverani E, Scaioli E, Cardamone C, Dal Monte P, Belluzzi A. Mycobacterium avium subspecies paratuberculosis in the etiology of Crohn's disease, cause or epiphenomenon? World J Gastroenterol. 2014 Sep;20(36):13060-70. 
16 Taurog JD, Richardson JA, Croft JT, Simmons WA, Zhou M, Fernández-Sueiro JL, et al. The germfree state prevents development of gut and joint inflammatory disease in HLA-B27 transgenic rats. J Exp Med. 1994 Dec;180(6):2359-64.

17 Hoentjen F, Harmsen HJ, Braat H, Torrice CD, Mann BA, Sartor RB, et al. Antibiotics with a selective aerobic or anaerobic spectrum have different therapeutic activities in various regions of the colon in interleukin 10 gene deficient mice. Gut. 2003 Dec;52(12):1721-7.

18 Rutgeerts P, Goboes K, Peeters M, Hiele M, Penninckx F, Aerts R, et al. Effect of faecal stream diversion on recurrence of Crohn's disease in the neoterminal ileum. Lancet. 1991 Sep;338(8770):771-4.

19 Harper PH, Lee EC, Kettlewell MG, Bennett MK, Jewell DP. Role of the faecal stream in the maintenance of Crohn's colitis. Gut. 1985 Mar;26(3):279-84.

20 Prantera C, Lochs H, Grimaldi M, Danese S, Scribano ML, Gionchetti P; Retic Study Group (Rifaximin-Eir Treatment in Crohn's Disease). Rifaximin-extended intestinal release induces remission in patients with moderately active Crohn's disease. Gastroenterology. 2012 Mar;142(3):473-481.e4.

21 Faith JJ, Guruge JL, Charbonneau M, Subramanian S, Seedorf H, Goodman AL, et al. The long-term stability of the human gut microbiota. Science. 2013 Jul;341(6141):1237439.

22 Seksik P, Rigottier-Gois L, Gramet G, Sutren M, Pochart P, Marteau P, et al. Alterations of the dominant faecal bacterial groups in patients with Crohn's disease of the colon. Gut. 2003 Feb;52(2):237-42.

23 Scanlan PD, Shanahan F, O’Mahony C, Marchesi JR. Culture-independent analyses of temporal variation of the dominant fecal microbiota and targeted bacterial subgroups in Crohn's disease. J Clin Microbiol. 2006 Nov; 44(11):3980-8.

24 Walters WA, Xu Z, Knight R. Meta-analyses of human gut microbes associated with obesity and IBD. FEBS Lett. 2014 Nov;588(22): 4223-33.

25 Manichanh C, Rigottier-Gois L, Bonnaud E, Gloux K, Pelletier E, Frangeul L, et al. Reduced diversity of faecal microbiota in Crohn's disease revealed by a metagenomic approach. Gut. 2006 Feb;55(2):205-11.

26 Frank DN, St Amand AL, Feldman RA, Boedeker EC, Harpaz N, Pace NR. Molecularphylogenetic characterization of microbial community imbalances in human inflammatory bowel diseases. Proc Natl Acad Sci USA. 2007 Aug;104(34):13780-5.

27 McLaughlin SD, Walker AW, Churcher C, Clark SK, Tekkis PP, Johnson MW, et al. The bacteriology of pouchitis: a molecular phylogenetic analysis using 16S rRNA gene cloning and sequencing. Ann Surg. 2010 Jul;252(1): $90-8$.

28 Gevers D, Kugathasan SZ, Denson LA, Vázquez-Baeza Y, Van Treuren W, Ren B, et al. The treatment-naive microbiome in newonset Crohn's disease. Cell Host and Microbe. 2014 Mar;15(3):382-92.
29 Joossens M, Huys G, Cnockaert M, De Preter V, Verbeke K, Rutgeerts P, et al. Dysbiosis of the faecal microbiota in patients with Crohn's disease and their unaffected relatives. Gut. 2011 May;60(5):631-7.

30 Mathewson ND, Jenq R, Mathew AV, Koenigsknecht M, Hanash A, Toubai T, et al. Gut microbiome-derived metabolites modulate intestinal epithelial cell damage and mitigate graft-versus-host disease. Nat Immunol. 2016 May; 17(5):505-13.

31 Machiels K, Joossens M, Sabino J, De Preter V, Arijs I, Eeckhaut V, et al. A decrease of the butyrate-producing species Roseburia hominis and Faecalibacterium prausnitzii defines dysbiosis in patients with ulcerative colitis. Gut. 2014 Aug;63(8):1275-83.

32 Couturier-Maillard A, Secher T, Rehman A, Normand S, De Arcangelis A, Haesler R, et al. NOD2-mediated dysbiosis predisposes mice to transmissible colitis and colorectal cancer. J Clin Invest. 2013 Feb;123(2):700-11.

33 Sellon RK, Tonkonogy S, Schultz M, Dieleman LA, Grenther W, Balish E, et al. Resident enteric bacteria are necessary for development of spontaneous colitis and immune system activation in interleukin-10-deficient mice. Infect Immun. 1998 Nov;66(11):522431.

34 Rehman A, Sina C, Gavrilova O, Häsler R, Ott S, Baines JF, et al. Nod2 is essential for temporal development of intestinal microbial communities. Gut. 2011 Oct;60(10):1354-62.

35 Sadaghian Sadabad M, Regeling A, de Goffau MC, Blokzijl T, Weersma RK, Penders J, et al. The ATG16L1-T300A allele impairs clearance of pathosymbionts in the inflamed ileal mucosa of Crohn's disease patients. Gut. 2015 Oct;64(10):1546-52.

36 Rausch P, Rehman A, Künzel S, Häsler R, Ott SJ, Schreiber S, et al. Colonic mucosa-associated microbiota is influenced by an interaction of Crohn disease and FUT2 (Secretor) genotype. Proc Natl Acad Sci USA. 2011 Nov; 108(47):19030-5.

37 Petnicki-Ocwieja T, Hrncir T, Liu YJ, Biswas A, Hudcovic T, Tlaskalova-Hogenova $\mathrm{H}$, et al. Nod2 is required for the regulation of commensal microbiota in the intestine. Proc Natl Acad Sci USA. 2009 Sep;106(37):15813-8.

38 Jiang W, Wang X, Zeng B, Liu L, Tardivel A, Wei $\mathrm{H}$, et al. Recognition of gut microbiota by NOD2 is essential for the homeostasis of intestinal intraepithelial lymphocytes. J Exp Med. 2013 Oct;210(11):2465-76.

39 Kim D, Kim YG, Seo SU, Kim DJ, Kamada N, Prescott D, et al. Nod2-mediated recognition of the microbiota is critical for mucosal adjuvant activity of cholera toxin. Nat Med. 2016 May;22(5):524-30.

40 Aden K, Rehman A, Falk-Paulsen M, Secher T, Kuiper J, Tran F, et al. Epithelial IL-23R Signaling Licenses Protective IL-22 Responses in Intestinal Inflammation. Cell Rep. 2016 Aug;16(8):2208-18.

41 Tschurtschenthaler M, Adolph TE, Ashcroft JW, Niederreiter L, Bharti R, Saveljeva S, et al. Defective ATG16L1-mediated removal of IRE1 a drives Crohn's disease-like ileitis. J Exp Med. 2017 Feb;214(2):401-22.
42 Litvak Y, Byndloss MX, Bäumler AJ. Colonocyte metabolism shapes the gut microbiota. Science. 2018 Nov;362(6418):eaat9076.

43 Wahlström A, Sayin SI, Marschall HU, Bäckhed F. Intestinal Crosstalk between Bile Acids and Microbiota and Its Impact on Host Metabolism. Cell Metab. 2016 Jul;24(1):41-50.

44 Wang Z, Klipfell E, Bennett BJ, Koeth R, Levison BS, Dugar B, et al. Gut flora metabolism of phosphatidylcholine promotes cardiovascular disease. Nature. 2011 Apr;472(7341): 57-63.

45 Hyams JS, Davis Thomas S, Gotman N, Haberman Y, Karns R, Schirmer M, et al. Clinical and biological predictors of response to standardised paediatric colitis therapy (PROTECT): a multicentre inception cohort study. Lancet. 2019 Apr;393(10182):1708-20.

46 Yilmaz B, Juillerat P, Øyås O, Ramon C, Bravo FD, Franc Y, et al.; Swiss IBD Cohort Investigators. Microbial network disturbances in relapsing refractory Crohn's disease. Nat Med. 2019 Feb;25(2):323-36.

47 Aden K, Rehman A, Waschina S, Pan WH, Walker A, Lucio M, et al. Metabolic Functions of Gut Microbes Associate With Efficacy of Tumor Necrosis Factor Antagonists in $\mathrm{Pa}$ tients With Inflammatory Bowel Diseases. Gastroenterology. 2019 Nov;157(5):1279-92.

48 Zou J, Chassaing B, Singh V, Pellizzon M, Ricci M, Fythe MD, et al. Fiber-Mediated Nourishment of Gut Microbiota Protects against Diet-Induced Obesity by Restoring IL-22Mediated Colonic Health. Cell Host Microbe. 2018 Jan;23(1):41-53.e4.

49 Hernández PP, Mahlakoiv T, Yang I, Schwierzeck V, Nguyen N, Guendel F, et al. Interferon- $\lambda$ and interleukin 22 act synergistically for the induction of interferon-stimulated genes and control of rotavirus infection. Nat Immunol. 2015 Jul;16(7):698-707.

50 Aden K, Tran F, Ito G, Sheibani-Tezerji R, Lipinski S, Kuiper JW, et al. ATG16L1 orchestrates interleukin-22 signaling in the intestinal epithelium via cGAS-STING. J Exp Med. 2018 Nov;215(11):2868-86.

51 Zhu W, Winter MG, Byndloss MX, Spiga L, Duerkop BA, Hughes ER, et al. Precision editing of the gut microbiota ameliorates colitis. Nature. 2018 Jan;553(7687):208-11.

52 Paramsothy S, Kamm MA, Kaakoush NO, Walsh AJ, van den Bogaerde J, Samuel D, et al. Multidonor intensive faecal microbiota transplantation for active ulcerative colitis: a randomised placebo-controlled trial. Lancet. 2017 Mar;389(10075):1218-28.

53 Costello SP, Hughes PA, Waters O, Bryant $\mathrm{RV}$, Vincent AD, Blatchford $\mathrm{P}$, et al. Effect of Fecal Microbiota Transplantation on 8-Week Remission in Patients With Ulcerative Colitis: A Randomized Clinical Trial. JAMA. 2019 Jan;321(2):156-64.

54 Paramsothy S, Nielsen S, Kamm MA, Deshpande NP, Faith JJ, Clemente JC, et al. Specific Bacteria and Metabolites Associated With Response to Fecal Microbiota Transplantation in Patients With Ulcerative Colitis. Gastroenterology. 2019 Apr;156(5):14401454.e2. 